\title{
Quantum strategies are better than classical in almost any XOR game*
}

\author{
Andris Ambainis, Artūrs Bačkurs, Kaspars Balodis, \\ Dmitrijs Kravčenko, Raitis Ozols, Juris Smotrovs, Madars Virza \\ Faculty of Computing, University of Latvia, \\ Raiņa bulv. 19, Rīga, LV-1586, Latvia
}

\begin{abstract}
We initiate a study of random instances of nonlocal games. We show that quantum strategies are better than classical for almost any 2-player XOR game. More precisely, for large $n$, the entangled value of a random 2-player XOR game with $n$ questions to every player is at least $1.21 \ldots$ times the classical value, for $1-o(1)$ fraction of all 2-player XOR games.
\end{abstract}

\section{Introduction}

Quantum mechanics is strikingly different from classical physics. In the area of information processing, this difference can be seen through quantum algorithms which can be exponentially faster than conventional algorithms [26, 24] and through quantum cryptography which offers degree of security that is impossible classically [5].

Another information-theoretic way of seeing the difference between quantum mechanics and the classical world is through non-local games. An example of a non-local game is the CHSH (Clauser-Horne-Shimony-Holt) game 9]. This is a game played by two players against a referee. The two players cannot communicate but can share common randomness or a common quantum state that is prepared before the beginning of the game. The referee sends an independent uniformly random bit to each of the two players. Each player responds by sending one bit back to the referee. Players win if $x \oplus y=i \wedge j$ where $i, j$ are the bits that the referee sent to the player and $x, y$ are players' responses. The maximum winning probability that can be achieved is 0.75 classically and $\frac{1}{2}+\frac{1}{2 \sqrt{2}}=0.85 \ldots$ quantumly.

There are several reasons why non-local games are interesting. First, CHSH game provides a very simple example to test the validity of quantum mechanics.

${ }^{*}$ Supported by ESF project 1DP/1.1.1.2.0/09/APIA/VIAA/044, FP7 Marie Curie International Reintegration Grant PIRG02-GA-2007-224886 and FP7 FET-Open project QCS. 
If we have implemented the referee and the two players $A, B$ by devices so that there is no communication possible between $A$ and $B$ and we observe the winning probability of $0.85 \ldots$, there is no classical explanation possible. Second, non-local games have been used in device-independent cryptography [1, 25].

Some non-local games show big gaps between the classical and the quantum winning probabilities. For example, Buhrman et al. [7] construct a 2player quantum game where the referee and the players send values $x, y, i, j \in$ $\{1, \ldots, n\}$ and the classical winning probability is $\frac{1}{2}+\Theta\left(\frac{1}{\sqrt{n}}\right)$ while the quantum winning probability is 1 . In contrast, Almeida et al. 2] construct a non-trivial example of a game in which quantum strategies provide no advantage at all.

Which of those is the typical behaviour? In this paper, we study this question by looking at random instances of non-local games.

More specifically, we study two-party XOR games. This is a subclass of non-local games with 2 players, where the referee randomly chooses inputs $i \in\{1,2, \ldots, n\}, j \in\{1,2, \ldots, k\}$ and sends them to the players. The players reply by sending bits $x$ and $y$. The rules of the game are specified by an $n \times k$ matrix $A$ whose entries are +1 and -1 . To win, the players must produce $x$ and $y$ with $x=y$ if $A_{i j}=1$ and $x$ and $y$ with $x \neq y$ if $A_{i j}=-1$.

We consider the case when the matrix $A$ that specifies the rules of the game is chosen randomly against all \pm 1 -valued $n \times k$ matrices $A$. For the case when $n=k$, we show that

- The maximum winning probability $p_{q}$ that can be achieved by a quantum strategy is $\frac{1}{2}+\frac{1 \pm o(1)}{\sqrt{n}}$ with a probability $1-o(1)$;

- The maximum winning probability $p_{c l}$ that can be achieved by a classical strategy satisfies

$$
\frac{1}{2}+\frac{0.6394 \ldots-o(1)}{\sqrt{n}} \leq p_{c l} \leq \frac{1}{2}+\frac{0.8325 \ldots+o(1)}{\sqrt{n}}
$$

with a probability $1-o(1)$.

In the literature on non-local games, one typically studies the difference between the winning probability $p_{q}\left(p_{c l}\right)$ and the losing probability $1-p_{q}\left(1-p_{c l}\right)$ : $\Delta_{q}=2 p_{q}-1\left(\Delta_{c l}=2 p_{c l}-1\right)$. The advantage of quantum strategies is then evaluated by the ratio $\frac{\Delta_{q}}{\Delta_{c l}}$. For random XOR games, our results imply that

$$
1.2011 \ldots<\frac{\Delta_{q}}{\Delta_{c l}}<1.5638 \ldots
$$

for almost all games. Our computer experiments suggest that, for large $n$, $\frac{\Delta_{q}}{\Delta_{c l}} \approx 1.305 \ldots$. For comparison, the biggest advantage that can be achieved in any 2-player XOR game is equal to Grothendieck's constant $K_{G}$ [14 about which we know that [16, 22, 6]

$$
1.67696 \ldots . \leq K_{G} \leq 1.7822139781 \ldots
$$

Thus, the quantum advantage in random XOR games is comparable to the maximum possible advantage for this class of non-local games. 
We find this result quite surprising. Quantum-over-classical advantage usually makes use of a structure that is present in the computational problem (such as the algebraic structure that enables Shor's quantum algorithm for factoring [24). Such structure is normally not present in random computational problems.

The methods that we use to prove our results are also quite interesting. The upper bounds are easy in both classical and quantum case but both lower bounds are fairly sophisticated. The lower bound on the classical value of random XOR games requires a subtle argument that reduces lower-bounding the classical value to analyzing a certain random walk. The lower bound for the entangled value requires proving a new version of Marčenko-Pastur law [18. for random matrices.

Related work. Junge and Palazuelos [17] have constructed non-local games with a big gap between the quantum (entangled) value and the classical value, via randomized constructions. The difference between this paper and [17] is as follows. The goal of [17] was to construct a big gap between the entangled value and the classical value of a non-local game and the probability distribution on non-local games and inputs was chosen so that this goal would be achieved.

Our goal is to study the behaviour of non-local games in the case when the conditions are random. We therefore choose a natural probability distribution on non-local games (without the goal of optimizing the quantum advantage) and study it. The surprising fact is that a substantial quantum advantage still exists in such setting.

\section{Technical preliminaries}

We use $[n]$ to denote the set $\{1,2, \ldots, n\}$.

In a 2-player XOR game, we have two players $A$ and $B$ playing against a referee. Players $A$ and $B$ cannot communicate but can share common random bits (in the classical case) or an entangled quantum state (in the quantum case). The referee randomly chooses values $i \in\{1, \ldots, n\}$ and $j \in\{1, \ldots, n\}$ and sends them to $A$ and $B$, respectively. Players $A$ and $B$ respond by sending answers $x \in\{0,1\}$ and $y \in\{0,1\}$ to the referee.

Players win if answers $x$ and $y$ satisfy some winning condition $P(i, j, x, y)$. For XOR games, the condition may only depend on the parity $x \oplus y$ of players' responses. Then, it can be written as $P(i, j, x \oplus y)$.

For this paper, we also assume that, for any $i, j$, exactly one of $P(i, j, 0)$ and $P(i, j, 1)$ is true. Then, we can describe a game by an $n \times n$ matrix $\left(A_{i j}\right)_{i, j=1}^{n}$ where $A_{i j}=1$ means that, given $i$ and $j$, players must output $x, y$ with $x \oplus y=0$ (equivalently, $x=y$ ) and $A_{i j}=-1$ means that players must output $x, y$ with $x \oplus y=1$ (equivalently, $x \neq y$ ).

Let $p_{S \text {,win }}$ be the probability that the players win if they use a strategy $S$ and $p_{S, l o s}=1-p_{S \text {,win }}$ be the probability that they lose. We will be interested in the difference $\Delta_{S}=p_{S, \text { win }}-p_{S, \text { los }}$ between the winning and the losing probabilities. The classical value of a game, $\Delta_{c l}$, is the maximum of $\Delta_{S}$ over all classical strategies $S$. The entangled value of a game, $\Delta_{q}$, is the maximum 
of $\Delta_{S}$ over all quantum strategies $S$.

Let $p_{i j}$ be the probability that the referee sends question $i$ to player $A$ and question $j$ to player $B$. Then [10, section 5.3], the classical value of the game is equal to

$$
\Delta_{c l}=\max _{u_{1}, \ldots, u_{n} \in\{-1,1\}} \max _{v_{1}, \ldots, v_{n} \in\{-1,1\}} \sum_{i, j=1}^{n} p_{i j} A_{i j} u_{i} v_{j} .
$$

In the quantum case, Tsirelson's theorem [8] implies that

$$
\Delta_{q}=\max _{u_{i}:\left\|u_{i}\right\|=1} \max _{v_{j}:\left\|v_{j}\right\|=1} \sum_{i, j=1}^{n} p_{i j} A_{i j}\left(u_{i}, v_{j}\right)
$$

where the maximization is over all tuples of unit-length vectors $u_{1}, \ldots, u_{n} \in R^{d}$, $v_{1}, \ldots, v_{n} \in R^{d}$ (in an arbitrary number of dimensions $d$ ).

We will assume that the probability distribution on the referee's questions $i, j$ is uniform: $p_{i j}=\frac{1}{n^{2}}$ and study $\Delta_{c l}$ and $\Delta_{q}$ for the case when $A$ is a random Bernoulli matrix (i.e., each entry $A_{i j}$ is +1 with probability $1 / 2$ and -1 with probability $1 / 2$, independently of other entries).

Other probability distributions on referee's questions can be considered, as well. For example, one could choose $y_{i j}$ to be normally distributed random variables with mean 0 and variance 1 and take $p_{i j}=\frac{\left|y_{i j}\right|}{\sum_{i, j=1}^{n}\left|y_{i j}\right|}$. Or, more generally, one could start with $y_{i j}$ being i.i.d. random variables from some arbitrary distribution $D$ and define $p_{i j}$ in a similar way.

Most of our results are still true in this more general setting (with mild assumptions on the probability distribution $D$ ). Namely, Theorem 1 and the upper bound part of Theorem 4 remain unchanged. The only exception is the lower bound part of Theorem 4 which relies on the fact that the probability distribution $p_{i j}$ is uniform. It might be possible to generalize our lower bound proof to other distributions $D$ but the exact constant in such generalization of our lower bound could depend on the probability distribution $D$.

\section{Quantum upper and lower bound}

Theorem 1 For a random 2-player XOR game with $n$ inputs for each player,

$$
\Delta_{q}=\frac{2 \pm o(1)}{\sqrt{n}}
$$

with probability $1-o(1)$.

Proof: Because of (2), proving our theorem is equivalent to showing that

$$
\max _{\left\|u_{i}\right\|=\left\|v_{j}\right\|=1} \sum_{i=1}^{n} \sum_{j=1}^{n} A_{i j}\left(u_{i}, v_{j}\right)=(2 \pm o(1)) n^{3 / 2}
$$

holds with probability $1-o(1)$. 
For the upper bound, we rewrite this expression as follows. Let $u$ be a vector obtained by concatenating all vectors $u_{i}$ and $v$ be a vector obtained by concatenating all $v_{j}$. Since $\left\|u_{i}\right\|=\left\|v_{j}\right\|=1$, we have $\|u\|=\|v\|=\sqrt{n}$. We have

$$
\sum_{i=1}^{n} \sum_{j=1}^{n} A_{i j}\left(u_{i}, v_{j}\right)=(u,(A \otimes I) v) \leq\|u\| \cdot\|A \otimes I\| \cdot\|v\| \leq\|A\| n .
$$

By known results on norms of random matrices 29, $\|A\|=(2+o(1)) \sqrt{n}$ with a high probability.

For the lower bound, we note that

$$
\max _{\left\|u_{i}\right\|=\left\|v_{j}\right\|=1} \sum_{i=1}^{n} \sum_{j=1}^{n} a_{i j}\left(u_{i}, v_{j}\right)=\max _{\left\|u_{i}\right\| \leq 1,\left\|v_{j}\right\| \leq 1} \sum_{i=1}^{n} \sum_{j=1}^{n} a_{i j}\left(u_{i}, v_{j}\right) .
$$

We have

Theorem 2 (Marčenko-Pastur law, [18]) Let $A$ be a $n \times n$ random matrix whose entries $A_{i j}$ are independent random variables with mean 0 and variance 1. Let $C \in[0,2]$. With probability $1-o(1)$, the number of singular values $\lambda$ of $A$ that satisfy $\lambda \geq C \sqrt{n}$ is $(f(C)-o(1)) n$ where

$$
f(C)=\frac{1}{2 \pi} \int_{x=C^{2}}^{4} \sqrt{\frac{4}{x}-1} d x .
$$

Let $\lambda_{1}, \ldots, \lambda_{m}$ be the singular values of $A$ that satisfy $\lambda_{i} \geq(2-\epsilon) \sqrt{n}$. With high probability, we have $m \in[(f(2-\epsilon)-o(1)) n,(f(2-\epsilon)+o(1)) n]$. We now assume that this is the case.

Let $l_{i}$ and $r_{i}$ be the corresponding left and right singular vectors: $A r_{i}=\lambda_{i} l_{i}$. (Here, we choose $l_{i}$ and $r_{i}$ so that $\left\|l_{i}\right\|=\left\|r_{i}\right\|=1$ for all $i$.) Let $l_{i j}$ and $r_{i j}$ be the components of $l_{i}$ and $r_{i}: l_{i}=\left(l_{i j}\right)_{j=1}^{n}$ and $r_{i}=\left(r_{i j}\right)_{j=1}^{n}$.

We define $u_{j}$ and $v_{j}$ in a following way:

$$
u_{j}=\left(l_{i j}\right)_{i=1}^{m}, \quad v_{j}=\left(r_{i j}\right)_{i=1}^{m} .
$$

We have

$$
\begin{aligned}
& \sum_{i=1}^{n} \sum_{j=1}^{n} a_{i j}\left(u_{i}, v_{j}\right)=\sum_{i=1}^{n} \sum_{j=1}^{n} \sum_{k=1}^{m} a_{i j} l_{k i} r_{k j} \\
& =\sum_{k=1}^{m}\left(l_{k}, A r_{k}\right)=\sum_{k=1}^{m} \lambda_{k} \geq(2-\epsilon) m \sqrt{n} .
\end{aligned}
$$

Since $\left\|l_{i}\right\|=\left\|r_{i}\right\|=1$ and the vectors $u_{i}$ and $v_{j}$ are obtained by rearranging the entries of $l_{i}$ and $r_{i}$, we have

$$
\sum_{i=1}^{n}\left\|u_{i}\right\|^{2}=\sum_{i=1}^{n}\left\|l_{i}\right\|^{2}=m
$$


and, similarly, $\sum_{i}\left\|v_{i}\right\|^{2}=m$. If $u_{i}$ and $v_{i}$ all were of the same length, we would have $\left\|u_{i}\right\|^{2}=\left\|v_{i}\right\|^{2}=\frac{m}{n}$. Then, replacing $u_{i}$ and $v_{i}$ by $u_{i}^{\prime}=\frac{u_{i}}{\left\|u_{i}\right\|}$ and $v_{i}^{\prime}=\frac{v_{i}}{\left\|v_{i}\right\|}$ would increase each vector $\sqrt{\frac{n}{m}}$ times and result in

$$
\sum_{i=1}^{n} \sum_{j=1}^{n} a_{i j}\left(u_{i}^{\prime}, v_{j}^{\prime}\right) \geq(2-\epsilon) n^{3 / 2} .
$$

To deal with the general case, we will show that almost all $u_{i}$ and $v_{i}$ are of roughly the same length. Then, a similar argument will be used. The key to our proof is a new modification of Marčenko-Pastur law.

Theorem 3 (Modified Marčenko-Pastur law) Let $A$ be an $n \times n$ random matrix whose entries $A_{i j}$ are independent random variables with mean 0 and variance 1. Let $C \in[0,2]$. Let $e_{i}$ be the $i^{\text {th }}$ vector of the standard basis. Let $P_{C}$ be the projector on the subspace spanned by the right singular vectors with singular values at least $C \sqrt{n}$. Then,

$$
\operatorname{Pr}\left[\left|\left\|P_{C} e_{i}\right\|^{2}-f(C)\right|>\epsilon\right]=O\left(\frac{1}{n}\right)
$$

with the big- $O$ constant depending on $C$ and $\epsilon$.

The same result also holds for the left singular vectors.

Proof: In appendix A.

We now complete the proof, assuming the modified Marčenko-Pastur law. Since $P_{C}$ is spanned by the right singular vectors $r_{1}, \ldots, r_{m}$, we have

$$
\left\|P_{C} e_{i}\right\|^{2}=\sum_{j=1}^{m}\left(r_{j}, e_{i}\right)^{2}=\sum_{j=1}^{m} r_{j i}^{2}=\left\|v_{i}\right\|^{2} .
$$

Therefore, the modified Marčenko-Pastur law means that

$$
\operatorname{Pr}\left[\left\|v_{i}\right\|^{2}>f(2-\epsilon)+\delta\right]=O\left(\frac{1}{n}\right) .
$$

Thus, the expected number of $i \in\{1, \ldots, n\}$ for which $\left\|v_{i}\right\|^{2}>f(2-\epsilon)+\delta$ is $O(1)$. We now apply the following transformations to vectors $v_{i}$ :

1. For each $v_{i}$ with $\left\|v_{i}\right\|^{2}>f(2-\epsilon)+\delta$ (or $u_{i}$ with $\left\|u_{i}\right\|^{2}>f(2-\epsilon)+\delta$ ), we replace it by the zero vector $\overrightarrow{0}$;

2. We replace each $v_{i}$ by

$$
v_{i}^{\prime}=\frac{v_{i}}{\sqrt{f(2-\epsilon)+\delta}}
$$

and similarly for $u_{i}$.

After the first step $\left\|v_{i}\right\|^{2} \leq f(2-\epsilon)+\delta$ for all $i$. Hence, after the second step, $\left\|v_{i}^{\prime}\right\|^{2} \leq 1$ for all $i$.

We now bound the effect of those two steps on the sum

$$
\sum_{i=1}^{n} \sum_{j=1}^{n} a_{i j}\left(u_{i}, v_{j}\right)
$$


Because of (3), the initial value of this sum is at least

$$
(2-\epsilon) m \sqrt{n} \geq(2-\epsilon)(f(2-\epsilon)-o(1)) n^{3 / 2} .
$$

Because of (4), $\left\|v_{j}\right\|^{2}=\left\|P_{C} e_{j}\right\|^{2} \leq\left\|e_{j}\right\|^{2}=1$. Similarly, $\left\|u_{i}\right\|^{2} \leq 1$. Hence, $\left|\left(u_{i}, v_{j}\right)\right| \leq 1$ and replacing one $v_{j}$ (or $u_{i}$ ) by 0 changes the sum by at most $\sum_{i=1}^{n}\left|a_{i j}\right|=n$. Replacing $O(1) v_{j}$ 's (or $u_{i}$ 's) changes it by $O(n)$. Since the sum (5) is of the order $\Theta\left(n^{3 / 2}\right)$, this is a lower order change.

Replacing $v_{i}$ 's by $v_{i}^{\prime}$ 's (and $u_{i}^{\prime}$ 's by similarly defined $u_{i}^{\prime}$ 's) increases each inner product $\left(u_{i}, v_{j}\right) \frac{1}{f(2-\epsilon)+\delta}$ times and achieves

$$
\sum_{i=1}^{n} \sum_{j=1}^{n} a_{i j}\left(u_{i}^{\prime}, v_{j}^{\prime}\right) \geq \frac{(2-\epsilon)(f(2-\epsilon)-o(1))}{f(2-\epsilon)+\delta} n^{3 / 2} .
$$

Since this can be achieved for any fixed $\epsilon>0$ and $\delta>0$, we get that

$$
\max _{\left\|u_{i}^{\prime}\right\| \leq 1,\left\|v_{j}^{\prime}\right\| \leq 1} \sum_{i=1}^{n} \sum_{j=1}^{n} a_{i j}\left(u_{i}^{\prime}, v_{j}^{\prime}\right) \geq(2-o(1)) n^{3 / 2} .
$$

\section{Classical upper and lower bound}

In the classical case, we have to estimate

$$
\max _{u_{1}, \ldots, u_{n} \in\{-1,1\}} \max _{v_{1}, \ldots, v_{n} \in\{-1,1\}} \sum_{i, j=1}^{n} A_{i j} u_{i} v_{j} .
$$

There are several ways how one can interpret this expression. First, (6) is equal to the $l_{\infty} \rightarrow l_{1}$ norm of $A$ (denoted $\|A\|_{\infty \rightarrow 1}$ ). It is known that, for a random matrix $A,\|A\|_{\infty \rightarrow 1}=\Theta(n \sqrt{n})$ (e. g., from [20]). but the exact constant under $\Theta$ is not known.

Gittens and Tropp [12] show that, if $A$ is a matrix whose entries are i.i.d. random variables, then

$$
\|A\|_{\infty \rightarrow 1} \leq 2 E\left(\|A\|_{c o l}+\left\|A^{T}\right\|_{c o l}\right)
$$

where $\|A\|_{c o l}$ denotes the sum of the $l_{2}$ norms of the columns of $A$. For our case, this gives an upper bound of $\|A\|_{\infty \rightarrow 1} \leq 4 n \sqrt{n}$ which is substantially weaker than our Theorem 4 below (and would be insufficient to show a gap between classical and entangled values for random XOR games).

In the context of statistical physics, there has been substantial work on determining the order of

$$
\max _{u_{1}, \ldots, u_{n} \in\{-1,1\}} \sum_{i, j=1}^{n} A_{i j} u_{i} u_{j}
$$

when $A_{i j}$ is a symmetric Gaussian matrix (each $A_{i j}=A_{j i}$ is an independent Gaussian random variable with mean 0 and variance 1 ). It is known that (7) is 
equal to $(1.527 \ldots+o(1)) n^{3 / 2}$ with probability $1-o(1)$. This was first discovered in 23, 21] and rigorously proven by Talagrand [28.

The quantities (6) and (7) are of similar flavour but are not identical and the work on (7) does not directly imply anything about our problem. b

One can also interpret (6) combinatorially, as a problem of "unbalancing lights" 3. In this interpretation, $n \times n$ matrix represents an array of lights, with each light being "on" $\left(A_{i j}=1\right)$ or "off" $\left(A_{i j}=-1\right)$. We are allowed to choose a row or a column and switch all lights in this row or column. The task is to maximize the difference between the number of lights that is on and the number of lights that is off. It is known that for any $n \times n$ matrix $A$ with \pm 1 entries, 6 is at least $\sqrt{\frac{2}{\pi}} n^{3 / 2}$ [3, p.19]. We were not able to find any work on evaluating (6) for a random matrix $A$ in this context.

Theorem 4 For a random 2-player XOR game, its classical value $\Delta_{c l}$ satisfies

$$
\frac{1.2789 \ldots}{\sqrt{n}} \leq \Delta_{c l} \leq \frac{2 \sqrt{\ln 2}+o(1)}{\sqrt{n}}=\frac{1.6651 \ldots+o(1)}{\sqrt{n}}
$$

with probability $1-o(1)$.

This is equivalent to

$$
1.2789 \ldots n^{3 / 2} \leq\|A\|_{\infty \rightarrow 1} \leq 1.6651 \ldots n^{3 / 2}
$$

for a Bernoulli random matrix $A$.

In computer experiments, the ratio $\frac{\|A\|_{\infty \rightarrow 1}}{n^{3 / 2}}$ grows with $n$ and reaches $1.4519 \ldots$ for $n=26$. By fitting a formula $a n^{3 / 2}+b n$ where the leading term is of the order $n^{3 / 2}$ and the largest correction term is of the order $n$ to the data, we obtained that

$$
\|A\|_{\infty \rightarrow 1} \approx 1.53274 \ldots n^{3 / 2}-0.472806 \ldots n .
$$

Figure 1 shows the fit. Curiously, the constant in front of $n^{3 / 2}$ is very close to the constant $1.527 \ldots$ for the sum (7). We are not sure whether this is a coincidence or there is some connection between the asymptotic behaviour of the two sums.

Proof: The upper bound follows straightforwardly from Chernoff bounds. We use the following form of Chernoff inequality:

Theorem 5 [3, p.263] Let $X_{1}, \ldots, X_{n}$ be independent random variables with $\operatorname{Pr}\left[X_{i}=1\right]=\operatorname{Pr}\left[X_{i}=-1\right]=\frac{1}{2}$ and let $X=X_{1}+\ldots+X_{n}$. Then,

$$
\operatorname{Pr}[X \geq a]<e^{-\frac{a^{2}}{2 n}} .
$$

Let $x_{1}, \ldots, x_{n} \in\{-1,1\}$ and $y_{1}, \ldots, y_{n} \in\{-1,1\}$ be arbitrary. If $A_{i j} \in$ $\{-1,1\}$ are uniformly random, then $A_{i j} x_{i} y_{j} \in\{-1,1\}$ are also uniformly random. Hence, $\sum_{i, j} A_{i j} x_{i} y_{j}$ is a sum of $n^{2}$ uniformly random values from $\{-1,1\}$. By Theorem 5

$$
\operatorname{Pr}\left[\sum_{i, j} A_{i j} x_{i} y_{j}>C n^{\frac{3}{2}}\right]<e^{\frac{-\left(C n^{\frac{3}{2}}\right)^{2}}{2 n^{2}}}=\frac{1}{e^{\frac{C^{2} n}{2}}} .
$$




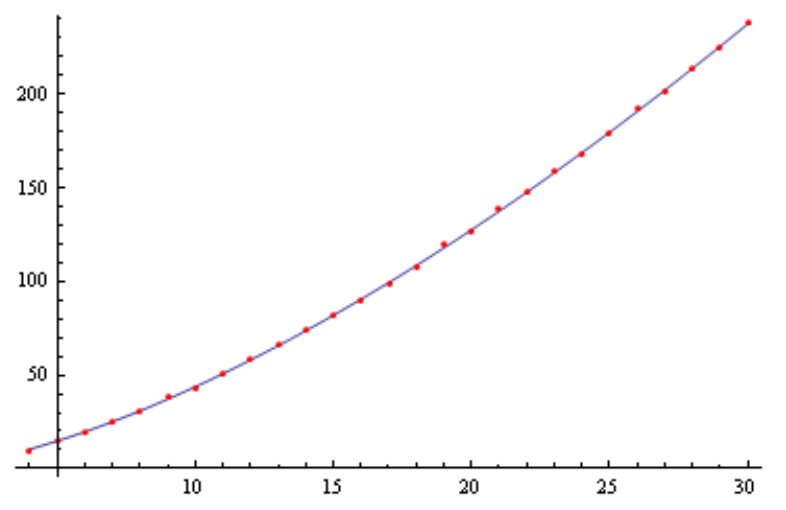

Figure 1: $\|A\|_{\infty \rightarrow 1}$, for random $n \times n$ matrices $A$

By taking $C=2 \sqrt{\ln 2}+2 \frac{\sqrt{\ln n}}{\sqrt{n}}$, we can ensure that this probability is less than $\frac{1}{2^{2 n} n^{2}}$. Then, by the union bound, the probability that $\sum_{i, j} A_{i j} x_{i} y_{j}>C n^{\frac{3}{2}}$ for some choice of $x_{i}$ 's and $y_{j}$ 's is less than $2^{2 n} \frac{1}{2^{2 n} n^{2}}=\frac{1}{n^{2}}$.

To prove the lower bound, we first show

Lemma 1 Let $A$ be an $n \times n$ random Bernoulli matrix. Then,

$$
\mathrm{E}_{A}\left[\max _{u_{i}, v_{j} \in\{-1,1\}} \sum_{i, j} u_{i} v_{j} A_{i j}\right] \geq(1.2789 \ldots-o(1)) n^{3 / 2} .
$$

Let $X=\max _{u_{i}, v_{j} \in\{-1,1\}} \sum_{i, j} u_{i} v_{j} A_{i j}$. By Lemma $1, E[X] \geq(1.2789 \ldots-$ $o(1)) n^{3 / 2}$. To prove that $X \geq(1.2789 \ldots-o(1)) n^{3 / 2}$ with probability $1-o(1)$, we show that $X$ is concentrated around $E[X]$.

Lemma 2 Let $X=\max _{u_{i}, v_{j} \in\{-1,1\}} \sum_{i, j} u_{i} v_{j} A_{i j}$ for a random $n \times n$ matrix A. Then,

$$
\operatorname{Pr}[|X-E[X]| \geq a n]<2 e^{-a^{2} / 8} .
$$

We then apply Lemma 2 with $a=\log n$ (or with $a=f(n)$ for any other $f(n)$ that has $f(n) \rightarrow \infty$ when $n \rightarrow \infty$ and $f(n)=o(\sqrt{n}))$ and combine it with Lemma 1 .

It remains to prove the two lemmas.

Proof: [of Lemma 1] Let $A$ be a random \pm 1 matrix. We choose $u_{i}$ and $v_{j}$, according to Algorithm 1

Because of the last step, we get that

$$
\sum_{i=1}^{n} \sum_{j=1}^{n} u_{i} v_{j} A_{i j}=\sum_{j=1}^{n}\left|S_{n, j}\right| .
$$

Each of $S_{n, j}$ is a random variable with an identical distribution. Hence,

$$
E\left[\sum_{i=1}^{n} \sum_{j=1}^{n} u_{i} v_{j} A_{i j}\right]=\sum_{j=1}^{n} E\left|S_{n, j}\right|=n E\left|S_{n, 1}\right| .
$$


1. Set $u_{1}=1$.

2. For each $k=2, \ldots, n$ do:

(a) For each $j=1, \ldots, n$, compute $S_{k-1, j}=\sum_{i=1}^{k-1} A_{i j} u_{i}$.

(b) Let $a_{k}=\left(Z\left(S_{k-1,1}\right), Z\left(S_{k-1,2}\right), \ldots, Z\left(S_{k-1, n}\right)\right)$ where $Z(x)=1$ if $x>0$, $Z(x)=-1$ if $x<0$ and $Z(x)=1$ or $Z(x)=-1$ with equal probability $\frac{1}{2}$ if $x=0$.

(c) Let $b_{k}=\left(A_{k 1}, A_{k 2}, \ldots, A_{k n}\right)$.

(d) Let $u_{i} \in\{+1,-1\}$ be such that $a_{i}$ and $b_{i} u_{i}$ agree in the maximum number of positions.

3. For each $j=1, \ldots, n$, let $v_{j}$ be such that $v_{j} S_{n, j} \geq 0$ where $S_{n, j}=\sum_{i=1}^{n} A_{i j} u_{i}$.

\section{Algorithm 1: Algorithm for choosing $u_{i}$ and $v_{j}$ for a given matrix $A$.}

We now consider a random walk with a reflecting boundary. The random walk starts at position 0 . If it is at the position 0 , it always moves to the position 1. If it is at the position $i>0$, it moves to the position $i+1$ with probability $\frac{1}{2}+\frac{\epsilon}{2}$ and position $i-1$ with probability $\frac{1}{2}-\frac{\epsilon}{2}$. Let $K_{i}^{\epsilon}$ be the position of the walker after $i$ steps.

Lemma $3\left|S_{n, 1}\right|=K_{n}^{\epsilon}$ for some $\epsilon=(1+o(1)) \sqrt{\frac{2}{\pi n}}$.

Proof: $b_{i}=\left(A_{i 1}, \ldots, A_{i n}\right)$ is a vector consisting of random \pm 1 's that is independent of $a_{i}$. Hence, the expected number of agreements between $a_{i}$ and $b_{i} u_{i}$ is $\left(\frac{1}{2}+\frac{\epsilon}{2}\right) n$ where $\epsilon=(1+o(1)) \sqrt{\frac{2}{\pi n}}$ [3. p.21]. Moreover, the probability of $a_{i}$ and $b_{i} u_{i}$ agreeing in location $j$ is the same for all $j$.

Hence, if $\left|S_{i-1,1}\right|>0$, we have $\left|S_{i, 1}\right|=\left|S_{i-1,1}\right|+1$ with probability $\frac{1}{2}+\frac{\epsilon}{2}$ and $\left|S_{i, 1}\right|=\left|S_{i-1,1}\right|-1$ with probability $\frac{1}{2}-\frac{\epsilon}{2}$. If $\left|S_{i-1,1}\right|=0$, then we always have $\left|S_{i, 1}\right|=1$.

Lemma 4 For a random walk with a reflecting boundary and $\epsilon=\frac{\alpha}{\sqrt{n}}$, we have $E\left[K_{n}^{\epsilon}\right] \geq(f(\alpha)-o(1)) \sqrt{n}$ where

$$
f(\alpha)=\frac{1}{2}\left(e^{-\frac{\alpha^{2}}{2}} \sqrt{\frac{2}{\pi}}+\alpha+\left(\frac{1}{\alpha}+\alpha\right) \operatorname{Erf}\left(\frac{\alpha}{\sqrt{2}}\right)\right) .
$$

Proof: In appendix B

By combining (8) and Lemmas 3 and 4 the probability of winning minus the probability of losing in the classical case of a random XOR game is at least

$$
\begin{gathered}
f\left(\sqrt{\frac{2}{\pi}}\right) \sqrt{n} \cdot n \cdot \frac{1}{n^{2}}=\frac{2+2 e^{-1 / \pi}+(2+\pi) \operatorname{Erf}\left(\frac{1}{\sqrt{\pi}}\right)}{2 \sqrt{2 \pi}} n^{-\frac{1}{2}} \\
=1.2789076012442957 \ldots n^{-\frac{1}{2}} .
\end{gathered}
$$


Proof: [of Lemma 2] Let

$$
f\left(A_{11}, A_{12}, \ldots, A_{n n}\right)=\max _{u_{i}, v_{j} \in\{-1,1\}} \sum_{i, j} u_{i} v_{j} A_{i j} .
$$

Then, changing one $A_{i j}$ from +1 to -1 (or from -1 to +1 ) changes $\sum_{i, j} u_{i} v_{j} A_{i j}$ by at most 2 . This means that $f\left(A_{11}, \ldots, A_{n n}\right)$ changes by at most 2 as well. In other words, $f$ is 2-Lipschitz. By applying Azuma's inequality [19, p. 303-305] with $c=2, t=n^{2}, \lambda=\frac{a}{2}$, we get

$$
\operatorname{Pr}\left[\left|f\left(A_{11}, \ldots, A_{n n}\right)-E\left[f\left(A_{11}, \ldots, A_{n n}\right)\right]\right| \geq a n\right]<2 e^{-a^{2} / 8} .
$$

\section{Conclusion}

We showed that quantum strategies are better than classical for random instances of XOR games. We expect that similar results may be true for other classes of non-local games.

A possible difficulty with proving them is that the mathematical methods for analyzing other classes of non-local games are much less developed. There is a well developed mathematical framework for studying XOR games [8, 10, 30] which we used in our paper. But even with that, some of our proofs were quite involved. Proving a similar result for a less well-studied class of games would be even more difficult.

Acknowledgments. We thank Assaf Naor, Oded Regev and Stanislaw Szarek for useful comments and references to related work.

\section{References}

[1] A. Acin, N. Brunner, N. Gisin, S. Massar, S. Pironio, V. Scarani. Deviceindependent security of quantum cryptography against collective attacks. Physical Review Letters, 98:230501, 2007.

[2] M. L. Almeida, J.-D. Bancal, N. Brunner, A. Acin, N. Gisin, S. Pironio. Guess your neighbour's input: a multipartite non-local game with no quantum advantage. Physical Review Letters, 104:230404, 2010. Also arXiv:1003.3844

[3] N. Alon, J. Spencer. The Probabilistic Method, Wiley, 2000.

[4] Z. Bai, J. Silverstein. Spectral Analysis of Large Dimensional Random Matrices, Springer, 2010.

[5] C. H. Bennett and G. Brassard, Quantum Cryptography: Public key distribution and coin tossing, Proceedings of the IEEE International Conference on Computers, Systems, and Signal Processing, Bangalore, p. 175 (1984). 
[6] M. Braverman, K. Makarychev, Y. Makarychev, A. Naor. The Groethendieck constant is strictly smaller than Krivine's bound. Proceedings of FOCS'2011, to appear.

[7] H. Buhrman, O. Regev, G. Scarpa, R. de Wolf. Near-optimal and explicit Bell inequality violations. Proceedings of Complexity'2011, pp. 157-166. Also arXiv:1012.5403.

[8] B. Cirelson (Tsirelson). Quantum generalizations of Bells inequality. Letters in Mathematical Physics, 4:93-100, 1980.

[9] J. Clauser, M. Horne, A. Shimony, and R. Holt, Physical Review Letters, 23:880-884, 1969.

[10] R. Cleve, P. Höyer, B. Toner, J. Watrous. Consequences and limits of nonlocal strategies. Proceedings of CCC'2004, pages 236-249, 2004. Also quant-ph/0404076.

[11] K. Davidson and S. Szarek. Local operator theory, random matrices and Banach spaces. In "W.B. Johnson, J. Lindenstrauss (eds.), Handbook on the Geometry of Banach spaces", vol. 1, pp. 317-366, Elsevier, 2001.

[12] A. Gittens, J. Tropp. Error bounds for random matrix approximation schemes. arXiv:0911.4108.

[13] R.L. Graham, D.E. Knuth, O. Patashnik, Concrete Mathematics, 2nd ed., Addison-Wesley, Reading, MA, 1994.

[14] A. Grothendieck. Resume de la theorie metrique des produits tensoriels topologiques. Boletim Sociedade De Matematico de Sao Paulo, 8:1-79, 1953.

[15] Lov K. Grover. A fast quantum mechanical algorithm for database search. Proceedings of STOC'1996, pp. 212-219.

[16] J.-L. Krivine. Sur la constante de Grothendieck. Comptes Rendus de l'Acadmie des Sciences, Series A-B, 284:A445-A446, 1977.

[17] M. Junge, C. Palazuelos. Large violation of Bell inequalities with low entanglement Communications in Mathematical Physics, 306 (3):695-746, 2011. arXiv:1007.3043.

[18] V. A. Marčenko and L. A. Pastur. Distribution of eigenvalues for some sets of random matrices. Math. USSR Sbornik, 1:457-483, 1967.

[19] M. Mitzenmacher, E. Upfal. Probability and Computing. Randomized Algorithms and Their Analysis. Cambridge University Press, 2005.

[20] A. M. Montero, A. M. Tonge. The Schur multiplication in tensor algebras. Studia Math., 68(1):1-24, 1980.

[21] G. Parisi. The order parameter for spin glasses: a function on the interval 01. Journal of Physics A: Mathemathical and General, 13:1101-1112, 1980.

$[22]$ J. A. Reeds. A new lower bound on the real Grothendieck constant. Unpublished manuscript, available at http://www.dtc.umn.edu/reedsj/bound2.dvi, 1991.

[23] D. Sherrington, S. Kirkpatrick. Infinite ranged models of spin glasses. Physical Review B, 17:4384-4403, 1978. 
[24] P. W. Shor. Algorithms for quantum computation: Discrete logarithms and factoring. In FOCS'1994, pages 124-134. IEEE.

[25] J. Silman, A. Chailloux, N. Aharon, I. Kerenidis, S. Pironio, S. Massar. Fully distrustful quantum cryptography. Physical Review Letters, 106:220501, 2011.

[26] D. R. Simon. On the power of quantum computation, In FOCS'1994, pages 116-123. IEEE.

[27] R. Stanley. Enumerative Combinatorics, vol. 2. Cambridge University Press, 1999.

[28] M. Talagrand. The generalized Parisi formula. Comptes Rendus de l'Acadmie des Sciences, Series I, 337:111-114, 2003.

[29] T. Tao. Topics in Random Matrix Theory. Draft of a book, available at http://terrytao.files.wordpress.com/2011/02/matrix-book.pdf

[30] S. Wehner. Tsirelson bounds for generalized Clauser-Horne-Shimony-Holt inequalities. Physical Review A, 73:022110, 2006. 


\section{A Modified Marčenko-Pastur law}

In this section, we prove Theorem 3 .

Without loss of generality, we assume that $i=1$. Let $\lambda_{1}, \ldots, \lambda_{n}$ be the singular values of $A$ and let $r_{i}$ be the corresponding right singular vectors. Let $\alpha_{i}=\left\langle e_{1} \mid r_{i}\right\rangle$.

Let $X$ be a random variable that is equal to $\frac{\lambda_{i}^{2}}{n}$ with probability $\left|\alpha_{i}\right|^{2}$. Let $Y$ be a random variable distributed according to the Marčenko-Pastur law with parameters $y=1$ and $\sigma=1$ [4, Section 3.1]. Then, the probability density function of $Y$ is equal to

$$
P(x)=\frac{1}{2 \pi} \sqrt{\frac{4}{x}-1}
$$

for $x \in[0,4]$ and $P(x)=0$ otherwise. Let $F_{X}(x)$ and $F_{Y}(x)$ denote the cumulative distribution functions of $X$ and $Y$ respectively. We would like to show that $F_{X}(x)$ and $F_{Y}(x)$ are close to one another.

We say that a family $\mathcal{X}$ of random variables is uniformly sub-Gaussian if there exist constants $c$ abd $C$ such that

$$
\operatorname{Pr}[|X|>x] \leq C e^{-c x^{2}}
$$

for every $X \in \mathcal{X}$. From Levy's continuity theorem [29, p. 99], we have

Theorem 6 Let $Y$ be a fixed random variable on some interval $[a, b]$ and let $\mathcal{X}$ be a uniformly sub-Gaussian family of random variables. Let $\epsilon>0$ be fixed. There exist $k>0, \delta>0$ such that if $X \in \mathcal{X}$ satisfies

$$
\left|E\left[X^{m}\right]-E\left[Y^{m}\right]\right| \leq \delta
$$

for all $m \in\{1, \ldots, k\}$, then we have

$$
\left|F_{X}(x)-F_{Y}(x)\right| \leq \epsilon
$$

for all $x \in[a, b]$.

We select a constant $D$ so that

$$
\operatorname{Pr}[\|A\| \geq D \sqrt{n}]=O\left(\frac{1}{n}\right) .
$$

By concentration results for norms of random matrices (Proposition 2.3.10 of [29]), $D=2+o(1)$ suffices. We define $\mathcal{X}$ as the family of random variables $X$, for all $A$ that satisfy $\|A\| \leq D \sqrt{n}$. Since $X=\frac{\lambda_{i}^{2}}{n}$ and $\lambda_{i} \leq\|A\|$, we have $\operatorname{Pr}\left[X>D^{2}\right]=0$. Hence, $\mathcal{X}$ is uniformly sub-Gaussian because constants $C, c$ can be chosen so that $C e^{-c x^{2}} \geq 1$ for $x: 0 \leq x \leq D^{2}$.

To show theorem 3 it suffices to prove that the random variable $X \in \mathcal{X}$ satisfies the condition 99 for all $m \in\{1,2, \ldots, k\}$, with probability $1-O\left(\frac{1}{n}\right)$. Since $k$ is fixed, this is equivalent to showing (9) for each $m \in\{1, \ldots, k\}$ with probability $1-O\left(\frac{1}{n}\right)$. For the random variable $Y$, its moments are quite well known: 


\section{Lemma 5}

$$
E\left[Y^{m}\right]=C_{m}
$$

where $C_{m}=\frac{(2 m) !}{m !(m+1) !}$ denotes the $m^{\text {th }}$ Catalan number.

Proof: From [4, Lemma 3.1], we have $E\left[Y^{m}\right]=\sum_{i=1}^{m} N(m, i)$ where $N(m, i)$ are Narayana numbers $N(m, i)=\frac{1}{i+1}\left(\begin{array}{c}m \\ i\end{array}\right)\left(\begin{array}{c}m-1 \\ i\end{array}\right)$. From [27, page 237], we have $\sum_{i=1}^{m} N(m, i)=C_{m}$

We introduce a random variable $M_{m}$ that is equal to $E\left[X^{m}\right]$ for a random matrix $A$. To show that $M_{m} \in\left[C_{m}-\delta, C_{m}+\delta\right]$ with a high probability, we bound the expectation and the variance of $M_{m}$ and then use Chebyshev's inequality. We have

Lemma 6 Let $m>0$ be fixed. Then, for large $n$,

$$
E\left[M_{m}\right]=\left(1+O\left(\frac{1}{n}\right)\right) C_{m}
$$

Proof: Let $B=A^{T} A$. Then, the eigenvalues of $B$ are $\lambda_{1}^{2}, \ldots, \lambda_{n}^{2}$. We have

$$
M_{m}=\frac{1}{n^{m}} \sum_{i=1}^{n} \lambda_{i}^{2 m}\left|\alpha_{i}\right|^{2}=\frac{1}{n^{m}}\left\langle e_{1}\left|B^{m}\right| e_{1}\right\rangle \text {. }
$$

By expanding $\left\langle e_{1}\left|B^{m}\right| e_{1}\right\rangle$, we can write

$$
M_{m}=\frac{1}{n^{m}} \sum_{i_{1}, \ldots, i_{2 m-1}=1}^{n} a_{i_{1}, 1} a_{i_{2 m-1}, 1} \prod_{j=1}^{m-1} a_{i_{2 j-1}, i_{2 j}} a_{i_{2 j+1}, i_{2 j}}
$$

$E\left[M_{m}\right]$ is equal to $\frac{1}{n^{m}}$ times the sum of expectations $E[T]$,

$$
T=a_{i_{1}, 1} a_{i_{2 m-1}, 1} \prod_{j=1}^{m-1} a_{i_{2 j-1}, i_{2 j}} a_{i_{2 j+1}, i_{2 j}} .
$$

If $T$ consists of $a_{j_{1}, k_{1}}, a_{j_{2}, k_{2}}, \ldots, a_{j_{l}, k_{l}}$ occurring $c_{1}, c_{2}, \ldots, c_{l}$ times, we have

$$
E[T]=E\left[a_{j_{1}, k_{1}}^{c_{1}}\right] E\left[a_{j_{2}, k_{2}}^{c_{2}}\right] \ldots E\left[a_{j_{l}, k_{l}}^{c_{l}}\right] .
$$

If $c_{i}$ is odd for some $i$, then $a_{j_{i}, k_{i}}^{c_{i}}$ is 1 with probability $1 / 2$ and -1 with probability $1 / 2$. Hence, $E\left[a_{j_{i}, k_{i}}^{c_{i}}\right]=0$. If all $c_{i}$ are even, then $a_{j_{i}, k_{i}}^{c_{i}}=1$ and 12 is equal to 1. Thus, 10 is equal to the number of terms $T$ in which each $a_{j_{i}, k_{i}}$ occurs an even number of times. We call such terms good.

For a term $T$, let $|T|$ to be the number of different numbers appearing in the sequence $1, i_{1}, i_{2}, \ldots, i_{2 m-1}$. We say that a good term $T$ is standard if the sequence $1, i_{1}, i_{2}, \ldots, i_{2 m-1}$ is such that the $i^{\text {th }}$ different number of this sequence is equal to $i$, for every $i \in\{2, \ldots,|T|\}$. If we have a standard term $T$, we can obtain $(n-1)(n-2) \ldots(n-|T|+1)$ good terms from it, by replacing all occurrences of $2, \ldots,|T|$ with distinct numbers $j_{2}, \ldots, j_{|T|} \in\{2, \ldots, n\}$. Moreover, each of those good terms can be obtained only from one standard term. 
Therefore, we have

$$
E\left[M_{m}\right]=\frac{1}{n^{m}} \sum_{T \text {-standard }}(n-1)(n-2) \ldots(n-|T|+1) .
$$

Since the sequence $1, i_{1}, i_{2}, \ldots, i_{2 m-1}$ may contain at most $2 m$ different numbers, we have $|T| \leq 2 m$. Since $m$ is a fixed constant, this implies

$$
E\left[M_{m}\right]=\frac{1}{n^{m}} \sum_{T-\text { standard }}\left(1+O\left(\frac{1}{n}\right)\right) n^{|T|-1} .
$$

Let $t$ be the largest possible value for $|T|$. Then,

$$
E\left[M_{m}\right]=\frac{1}{n^{m}}\left(1+O\left(\frac{1}{n}\right)\right) n^{t-1} \mid\{T: T \text { - standard, }|T|=t\} \mid .
$$

The contributions from standard terms $T$ with $|T|<t$ can be absorbed into the $O\left(\frac{1}{n}\right)$ factor, since $n^{|T|-1} \leq n^{t-2}=\frac{1}{n} n^{t-1}$ for each of those terms and the number of standard terms is a constant (it depends only on $t$ and not $n$ ).

It remains to determine $t$ and to count the standard terms $T$ with $|T|=t$. We claim that $t=m+1$.

Let $T$ be a standard term. Consider the graph $G$ with the set of vertices $V=\{1, \ldots,|T|\}$ and the set of edges $E$ consisting of all the different pairs $(i, j)$ that appear in the sequence $\left(i_{1}, 1\right),\left(i_{1}, i_{2}\right),\left(i_{3}, i_{2}\right), \ldots,\left(i_{2 m-1}, 1\right)$. Since $T$ is good, each such $(i, j)$ appears an even number of times, i.e. at least twice. Therefore, $|E| \leq \frac{2 m}{2}=m$.

The graph $G$ is connected. Hence, $|V| \leq|E|+1 \leq m+1$. Moreover, $|V|=m+1$ is achieved, for example, by the graph $G$ obtained from a standard term

$$
(2,1),(2,1),(3,1),(3,1), \ldots,(m+1,1),(m+1,1)
$$

in which $i_{2 j}=1$ and $i_{2 j-1}=j+1$. It remains to count the standard terms $T$ with $|T|=m+1$.

For each such term, we have $|V|=m+1$ and $|E|=m$. Hence, $G$ must be a tree, with each edge occuring exactly twice in the sequence $\left(i_{1}, 1\right),\left(i_{1}, i_{2}\right)$, $\left(i_{3}, i_{2}\right), \ldots,\left(i_{2 m-1}, 1\right)$. We now consider the sequence

$$
\left(1, i_{1}\right),\left(i_{1}, i_{2}\right),\left(i_{2}, i_{3}\right), \ldots,\left(i_{2 m-1}, 1\right)
$$

in which all edges are directed so that the sequence is a closed walk in the tree $G$. The conditions on this sequence that we have are the same as the conditions on a non-crossing cycle in [29, p. 144]. As shown in [29, p. 144-145], the number of non-crossing cycles of length $2 m$ is equal to the Catalan number $C_{m}$. The lemma follows by substituting this and $t=m+1$ into equation (13).

By a similar argument, we have

Lemma 7 Let $m>0$ be fixed. Then, for large $n$,

$$
E\left[M_{m}^{2}\right]=\left(1+O\left(\frac{1}{n}\right)\right)\left(C_{m}\right)^{2} .
$$


Proof: The proof is similar to the proof of Lemma 6. We omit it in this version of the paper.

From Lemmas 6 and 7 , we get the following corollaries.

Corollary 1 Let $m>0$ be fixed. Then, for large $n$,

$$
D\left[M_{m}\right]=O\left(\frac{1}{n}\right) C_{m}^{2}
$$

Proof: Follows from $D[X]=E\left[X^{2}\right]-E^{2}[X]$, Lemma 6 and Lemma 7 .

Corollary 2 Let $m>0$ and $\delta>0$ be fixed. Then, for large $n$,

$$
\operatorname{Pr}\left[\left|M_{m}-C_{m}\right| \geq \delta\right]=O\left(\frac{1}{n}\right) .
$$

Proof: Follows from Corollary 1 and Chebyshev inequality.

This completes the proof of Theorem 3 .

\section{B Analysis of a random walk with a reflecting boundary}

\section{B.1 Proof overview}

In this section, we prove Lemma 4. To simplify notation, we write $K_{i}$ instead of $K_{i}^{\epsilon}$. We have

$$
E\left[K_{i+1}\right]=\operatorname{Pr}\left[K_{i}>0\right] E\left[K_{i+1} \mid K_{i}>0\right]+\operatorname{Pr}\left[K_{i}=0\right] E\left[K_{i+1} \mid K_{i}=0\right] .
$$

If $K_{i}=m$ and $m>0$, we have

$$
E\left[K_{i+1} \mid K_{i}=m\right]=\left(\frac{1}{2}+\frac{\epsilon}{2}\right)(m+1)+\left(\frac{1}{2}-\frac{\epsilon}{2}\right)(m-1)=m+\epsilon .
$$

We also have $E\left[K_{i+1} \mid K_{i}=0\right]=1$. Hence,

$$
E\left[K_{i+1}\right]=E\left[K_{i}\right]+\epsilon+(1-\epsilon) \operatorname{Pr}\left[K_{i}=0\right] .
$$

By induction, this implies

$$
E\left[K_{n}\right]=\epsilon n+(1-\epsilon) \sum_{i=0}^{n-1} \operatorname{Pr}\left[K_{i}=0\right] .
$$

We have $\epsilon n=\alpha \sqrt{n}$. It remains to bound $\sum_{i=0}^{n-1} \operatorname{Pr}\left[K_{i}=0\right]$. The first step is to express this sum in terms of binomial coefficients.

\section{Claim 1}

$\sum_{i=0}^{n-1} \operatorname{Pr}\left[K_{i}=0\right]=1+\sum_{S=0}^{2\left\lfloor\frac{n-1}{2}\right\rfloor-2} \sum_{A=\max \left(0, S-\left\lfloor\frac{n-1}{2}\right\rfloor+1\right)}^{\left\lfloor\frac{S}{2}\right\rfloor}\left(\left(\begin{array}{c}S \\ A\end{array}\right)-\left(\begin{array}{c}S \\ A-1\end{array}\right)\right) p^{A}(1-p)^{S-A+1}$ where $p=\frac{1}{2}+\frac{\epsilon}{2}$. 
We can express this sum as a difference of two sums

$$
\begin{gathered}
\sum_{S=0}^{2\left\lfloor\frac{n-1}{2}\right\rfloor-2} \sum_{A=\max \left(0, S-\left\lfloor\frac{n-1}{2}\right\rfloor+1\right)}^{\left\lfloor\frac{S}{2}\right\rfloor}\left(\left(\begin{array}{c}
S \\
A
\end{array}\right)-\left(\begin{array}{c}
S \\
A-1
\end{array}\right)\right) p^{A}(1-p)^{S-A+1}= \\
(1-p) \sum_{S=0}^{2\left\lfloor\frac{n-1}{2}\right\rfloor-2} \sum_{A=0}^{\left\lfloor\frac{S}{2}\right\rfloor}\left(\left(\begin{array}{c}
S \\
A
\end{array}\right)-\left(\begin{array}{c}
S \\
A-1
\end{array}\right)\right) p^{A}(1-p)^{S-A} \\
-(1-p) \sum_{S=\left\lfloor\frac{n-1}{2}\right\rfloor} \sum_{A=0}^{2\left\lfloor\frac{n-1}{2}\right\rfloor-2}\left(\left(\begin{array}{c}
S \\
A
\end{array}\right)-\left(\begin{array}{c}
S \\
A-1
\end{array}\right)\right) p^{A}(1-p)^{S-A} .
\end{gathered}
$$

\section{Claim 2}

$$
\begin{gathered}
\sum_{S=0}^{2\left\lfloor\frac{n-1}{2}\right\rfloor-2} \sum_{A=0}^{\left\lfloor\frac{S}{2}\right\rfloor}\left(\left(\begin{array}{c}
S \\
A
\end{array}\right)-\left(\begin{array}{c}
S \\
A-1
\end{array}\right)\right) p^{A}(1-p)^{S-A} \geq \\
(1+o(1))\left(e^{-\frac{\alpha^{2}}{2}} \sqrt{\frac{2}{\pi}}-\alpha+\left(\frac{1}{\alpha}+\alpha\right) \operatorname{Erf}\left(\frac{\alpha}{\sqrt{2}}\right)\right) \sqrt{n}
\end{gathered}
$$

where

$$
\operatorname{Erf}(z)=\frac{2}{\sqrt{\pi}} \int_{0}^{z} e^{-t^{2}} d t .
$$

is the error function for the normal distribution.

\section{Claim 3}

$$
\sum_{S=\left\lfloor\frac{n-1}{2}\right\rfloor}^{2\left\lfloor\frac{n-1}{2}\right\rfloor-2} \sum_{A=0}^{S-\left\lfloor\frac{n-1}{2}\right\rfloor}\left(\left(\begin{array}{c}
S \\
A
\end{array}\right)-\left(\begin{array}{c}
S \\
A-1
\end{array}\right)\right) p^{A}(1-p)^{S-A}<1 .
$$

Lemma 4 now follows by combining Claims 1, 2 and 3 .

\section{B.2 Proof of Claim 1}

Let $T(m, t)$ be the number of paths of length $2 m$ that start and end at the point 0 , increase or decrease the coordinate by 1 at each step, never take a negative coordinate and return to the location $0 t$ times (including the last step). Then, for any $m>0$ and $t: 1 \leq t \leq m$, we have [13, p. 203],

$$
T(m, t)=\frac{t}{m}\left(\begin{array}{c}
2 m-t-1 \\
m-t
\end{array}\right)
$$

We have

$$
\sum_{i=0}^{n-1} \operatorname{Pr}\left[K_{i}=0\right]=1+\sum_{m=1}^{\left\lfloor\frac{n-1}{2}\right\rfloor} \operatorname{Pr}\left[K_{2 m}=0\right]=
$$




$$
\begin{gathered}
1+\sum_{m=1}^{\left\lfloor\frac{n-1}{2}\right\rfloor} \sum_{t=1}^{m} T(m, t) p^{m-t}(1-p)^{m}= \\
1+\sum_{m=1}^{\left\lfloor\frac{n-1}{2}\right\rfloor} \sum_{t=1}^{m}\left(\begin{array}{c}
2 m-t-1 \\
m-t
\end{array}\right) \frac{t}{m} p^{m-t}(1-p)^{m} .
\end{gathered}
$$

Here, the first equality follows from the fact that the random walk can return to location 0 only after an even number of steps. The second equality follows by partitioning the paths that return to 0 after $2 m$ steps according to the total number of times the path returns to 0 (including the final return to 0 ). If the path returns to $0 t$ times, then it also leaves $0 t$ times. Hence, there are $t$ steps in which we move right with probability 1 (the steps which start at location 0 ). There are also $m-t$ other steps when the path moves right (each of those steps is taken with probability $p$ ) and $m$ steps when the path moves left (each of those steps is taken with probability $1-p$ ). This means that the probability of each path with $t$ returns is $p^{m-t}(1-p)^{m}$. The third equality follows from (15).

Let $S=2 m-t-1$ and $A=m-t$. We can rewrite the sum (16) in the following way:

$$
\begin{gathered}
\sum_{m=1} \sum_{t=1}^{\left\lfloor\frac{n-1}{2}\right\rfloor}\left(\begin{array}{c}
2 m-t-1 \\
m-t
\end{array}\right) \frac{t}{m} p^{m-t}(1-p)^{m}= \\
\sum_{S=0}^{\left\lfloor\left\lfloor\frac{n-1}{2}\right\rfloor-2\right.} \sum_{A=\max \left(0, S-\left\lfloor\frac{n-1}{2}\right\rfloor+1\right)}^{\left\lfloor\frac{S}{2}\right\rfloor}\left(\begin{array}{c}
S \\
A
\end{array}\right) \frac{S-2 A+1}{S-A+1} p^{A}(1-p)^{S-A+1}= \\
\sum_{S=0} \sum_{A=\max \left(0, S-\left\lfloor\frac{n-1}{2}\right\rfloor-2\right.}^{\left\lfloor\frac{S}{2}\right\rfloor}\left(\left(\begin{array}{l}
S \\
A
\end{array}\right)-\left(\begin{array}{c}
S \\
A-1
\end{array}\right)\right) p^{A}(1-p)^{S-A+1}
\end{gathered}
$$

where the last equality follows from

$$
\left(\begin{array}{l}
S \\
A
\end{array}\right) \frac{S-2 A+1}{S-A+1}=\left(\begin{array}{l}
S \\
A
\end{array}\right)-\left(\begin{array}{c}
S \\
A-1
\end{array}\right) .
$$

This completes the proof of the claim.

\section{B.3 Proof of Claim 2}

We denote $m=2\left\lfloor\frac{n-1}{2}\right\rfloor-2$. By using the identity

$$
\begin{gathered}
\sum_{A=0}^{k}\left(\left(\begin{array}{l}
S \\
A
\end{array}\right)-\left(\begin{array}{c}
S \\
A-1
\end{array}\right)\right) p^{A}(1-p)^{S-A}= \\
\left(\begin{array}{l}
S \\
k
\end{array}\right) p^{k}(1-p)^{S-k}-\frac{2 \epsilon}{1-\epsilon} \sum_{A=0}^{k-1}\left(\begin{array}{l}
S \\
A
\end{array}\right) p^{A}(1-p)^{S-A},
\end{gathered}
$$


we obtain

$$
\begin{gathered}
\sum_{S=0}^{m} \sum_{A=0}^{\left\lfloor\frac{S}{2}\right\rfloor}\left(\left(\begin{array}{c}
S \\
A
\end{array}\right)-\left(\begin{array}{c}
S \\
A-1
\end{array}\right)\right) p^{A}(1-p)^{S-A}= \\
\sum_{S=0}^{m}\left(\begin{array}{c}
S \\
\left\lfloor\frac{S}{2}\right\rfloor
\end{array}\right) p^{\left\lfloor\frac{S}{2}\right\rfloor}(1-p)^{\left\lceil\frac{S}{2}\right\rceil}-2 \epsilon(1+o(1)) \sum_{S=1}^{m} \sum_{A=0}^{\left\lfloor\frac{S}{2}\right\rfloor-1}\left(\begin{array}{c}
S \\
A
\end{array}\right) p^{A}(1-p)^{S-A} .
\end{gathered}
$$

Claim 2 now follows from the following two claims.

\section{Claim 4}

$$
\sum_{S=0}^{m}\left(\begin{array}{c}
S \\
\left\lfloor\frac{S}{2}\right\rfloor
\end{array}\right) p^{\left\lfloor\frac{S}{2}\right\rfloor}(1-p)^{\left\lceil\frac{S}{2}\right\rceil} \geq(1+o(1)) \frac{2 \sqrt{n}}{\alpha} \operatorname{Erf}\left(\frac{\alpha}{\sqrt{2}}\right) .
$$

\section{Claim 5}

$2 \epsilon \sum_{S=1}^{m} \sum_{A=0}^{\left\lfloor\frac{S}{2}\right\rfloor-1}\left(\begin{array}{l}S \\ A\end{array}\right) p^{A}(1-p)^{S-A} \leq(1+o(1)) \sqrt{n}\left(\alpha-e^{-\frac{\alpha^{2}}{2}} \sqrt{\frac{2}{\pi}}+\left(\frac{1}{\alpha}-\alpha\right) \operatorname{Erf}\left(\frac{\alpha}{\sqrt{2}}\right)\right)$

Proof: [of Claim 4] We have

$$
\begin{gathered}
\left(\begin{array}{c}
S \\
\left\lfloor\frac{S}{2}\right\rfloor
\end{array}\right) p^{\left\lfloor\frac{S}{2}\right\rfloor}(1-p)^{\left\lceil\frac{S}{2}\right\rceil}=(1+o(1)) \frac{\sqrt{2}}{\sqrt{\pi S}} 2^{S}(p(1-p))^{S / 2} \\
=(1+o(1)) \frac{\sqrt{2}}{\sqrt{\pi S}} e^{-\gamma S}
\end{gathered}
$$

where $\gamma=\frac{-\ln 4 p(1-p)}{2}$. Here, the first equality follows from approximations of binomial coefficients. We denote $W(n, S)=\frac{\sqrt{2} e^{-\gamma S}}{\sqrt{\pi S}}$. The identity 18 means that, for every $\delta>0$, there exists $S_{0}$ such that

$$
\left(\begin{array}{c}
S \\
\left\lfloor\frac{S}{2}\right\rfloor
\end{array}\right) p^{\left\lfloor\frac{S}{2}\right\rfloor}(1-p)^{\left\lceil\frac{S}{2}\right\rceil} \geq(1-\delta) W(n, S)
$$

for all $S \geq S_{0}$. Therefore, we have

$$
\begin{gathered}
\sum_{S=0}^{m}\left(\begin{array}{c}
S \\
\left\lfloor\frac{S}{2}\right\rfloor
\end{array}\right) p^{\left\lfloor\frac{S}{2}\right\rfloor}(1-p)^{\left\lceil\frac{S}{2}\right\rceil} \geq(1-\delta) \sum_{S=S_{0}}^{m} W(n, S) \\
\geq(1-\delta) \int_{S_{0}}^{m} W(n, S) d S \\
=(1-\delta) \frac{\sqrt{2}}{\gamma}\left(\operatorname{Erf}(\sqrt{\gamma m})-\operatorname{Erf}\left(\sqrt{\gamma S_{0}}\right)\right)
\end{gathered}
$$

Here, the inequality follows from the function $W(n, S)$ being decreasing in $S$. The last equality follows from $\int \frac{e^{-a t}}{\sqrt{t}} d t=\frac{\sqrt{\pi} \operatorname{Erf}(\sqrt{a t})}{\sqrt{a}}$. For large $n$, we have

$$
\gamma=\frac{-\ln 4 p(1-p)}{2}=\frac{-\ln \left(1-\epsilon^{2}\right)}{2}=\frac{-\ln \left(1-\frac{\alpha^{2}}{n}\right)}{2}=(1+o(1)) \frac{\alpha^{2}}{2 n} .
$$


We also have $\gamma m=(1+o(1)) \frac{\alpha^{2} m}{2 n}=(1+o(1)) \frac{\alpha^{2}}{2}$ and $\gamma S_{0}=(1+o(1)) \frac{\alpha^{2} S_{0}}{2 n}=$ $o(1)$. Hence, 19) is equal to

$$
(1-\delta)(1+o(1)) \frac{2 \sqrt{n}}{\alpha} \operatorname{Erf}\left(\frac{\alpha}{\sqrt{2}}\right) .
$$

Since this is true for any $\delta>0$, the claim follows.

Proof: [of Claim 5] We can interpret $\sum_{A=0}^{\left\lfloor\frac{S}{2}-1\right\rfloor}\left(\begin{array}{l}S \\ A\end{array}\right) p^{A}(1-p)^{S-A}$ as follows. Let $X=X_{1}+\ldots+X_{S}$ where $X_{i}$ are independent random variables with $\operatorname{Pr}\left[X_{i}=1\right]=p$ and $\operatorname{Pr}\left[X_{i}=-1\right]=1-p$. Then,

$$
\sum_{A=0}^{\left\lfloor\frac{S}{2}\right\rfloor-1}\left(\begin{array}{l}
S \\
A
\end{array}\right) p^{A}(1-p)^{S-A}=\operatorname{Pr}\left[X \leq\left(\left\lfloor\frac{S}{2}\right\rfloor-1\right)-\left(S-\left\lfloor\frac{S}{2}\right\rfloor+1\right)\right] \leq \operatorname{Pr}[X \leq 0] .
$$

By Central limit theorem, for large $S, \operatorname{Pr}[X \leq 0]$ tends to

$$
\frac{1}{2} \operatorname{Erfc}\left(\frac{0-E[X]}{\sqrt{2 D[X]}}\right)=\frac{1}{2} \operatorname{Erfc}\left(\frac{(1-2 p) S}{\sqrt{4 p S}}\right)
$$

where $\operatorname{Erfc}(x)=1-\operatorname{Erf}(x)$. Since $p=\frac{1}{2}+\frac{\epsilon}{2}, \epsilon=\frac{\alpha}{\sqrt{n}}$, this is equal to

$$
\frac{1}{2} \operatorname{Erfc}\left(\frac{\sqrt{S} \epsilon}{\sqrt{2+\epsilon}}\right)=(1+o(1)) \frac{1}{2} \operatorname{Erfc}\left(\frac{\sqrt{S} \epsilon}{\sqrt{2}}\right) .
$$

Hence,

$$
\begin{gathered}
2 \epsilon \sum_{S=1}^{m} \sum_{A=0}^{\left\lfloor\frac{S}{2}\right\rfloor-1}\left(\begin{array}{l}
S \\
A
\end{array}\right) p^{A}(1-p)^{S-A}=(1+o(1)) \epsilon \sum_{S=1}^{m} \operatorname{Erfc}\left(\frac{\sqrt{S} \epsilon}{\sqrt{2}}\right) \\
<(1+o(1)) \frac{\alpha}{\sqrt{n}} \int_{0}^{n} \operatorname{Erfc}\left(\frac{\sqrt{S} \alpha}{\sqrt{2 n}}\right) d S \\
=(1+o(1)) \sqrt{n}\left(\alpha-e^{-\frac{\alpha^{2}}{2}} \sqrt{\frac{2}{\pi}}+\left(\frac{1}{\alpha}-\alpha\right) \operatorname{Erf}\left(\frac{\alpha}{\sqrt{2}}\right)\right) .
\end{gathered}
$$

Here, the inequality follows from Erfc being decreasing and $m<n$ and the last equality follows from

$$
\int \operatorname{Erfc}(a \sqrt{t}) d t=\frac{\operatorname{Erf}(a \sqrt{t})}{2 a^{2}}-\frac{\sqrt{t} e^{-a^{2} t}}{\sqrt{\pi} a}+t(1-\operatorname{Erf}(a \sqrt{t})) .
$$

\section{B.4 Proof of Claim 3}

Let $m=\left\lfloor\frac{n-1}{2}\right\rfloor$. Because of 17$)$, we have

$$
\sum_{S=m}^{2 m-2} \sum_{A=0}^{S-m}\left(\left(\begin{array}{l}
S \\
A
\end{array}\right)-\left(\begin{array}{c}
S \\
A-1
\end{array}\right)\right) p^{A}(1-p)^{S-A}
$$




$$
\begin{gathered}
=\sum_{S=m}^{2 m-2}\left(\begin{array}{l}
S \\
m
\end{array}\right) p^{S-m}(1-p)^{m}-\frac{2 \epsilon}{1-\epsilon} \sum_{S=m}^{2 m-2} \sum_{A=0}^{S-m-1}\left(\begin{array}{l}
S \\
A
\end{array}\right) p^{A}(1-p)^{S-A} \\
<\sum_{S=m}^{2 m-2}\left(\begin{array}{c}
S \\
m
\end{array}\right) 2^{-S}
\end{gathered}
$$

where the last inequality follows from

$$
p^{A}(1-p)^{S-A} \leq 2^{-S}
$$

that holds if $2 A \leq S$. Claim 3 now follows from

Claim 6 Let $m$ be a positive integer. Then,

$$
\sum_{S=m}^{2 m}\left(\begin{array}{c}
S \\
m
\end{array}\right) 2^{-S}=1
$$

Proof: We give a combinatorial proof of this equality.

Consider a random process where we have two boxes each containing $m+1$ pebbles. At each step we choose one of the two boxes with equal probability $\frac{1}{2}$ and remove one pebble from this box. The process finishes when we remove the last pebble from one of the two boxes.

We calculate

$$
\operatorname{Pr}[\text { The process finishes after } k \text { steps }]=
$$

$2 \operatorname{Pr}$ [The process finishes after $k$ steps and the first box is empty]

To empty the first box we need to choose the first box in $m$ out of $k-1$ steps which happens with a probability of $\left(\begin{array}{c}k-1 \\ m\end{array}\right) \frac{1}{2^{k-1}}$ and in the last step we need to choose the first box which happens with a probability of $\frac{1}{2}$. Thus

$$
\operatorname{Pr} \text { [The process finishes after } k \text { steps }]=2\left(\begin{array}{c}
k-1 \\
m
\end{array}\right) \frac{1}{2^{k-1}} \frac{1}{2}=\left(\begin{array}{c}
k-1 \\
m
\end{array}\right) \frac{1}{2^{k-1}} .
$$

The number of steps $k$ can be any value from $m+1$ to $2 m+1$ including. Because the process must end after some number of steps we obtain

$$
\sum_{k=m+1}^{2 m+1}\left(\begin{array}{c}
k-1 \\
m
\end{array}\right) \frac{1}{2^{k-1}}=1 .
$$

Claim 3 follows by substituting $k=S+1$. 